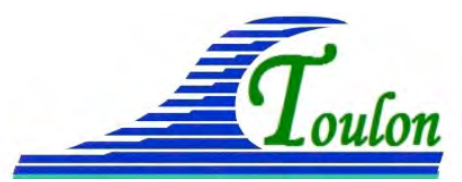
XIV èmes Journées Nationales Génie Côtier - Génie Civil
Toulon, 29 juin au $1^{\text {er }}$ juillet 2016

DOI:10.5150/jngcgc.2016.050 @ Editions Paralia CFL

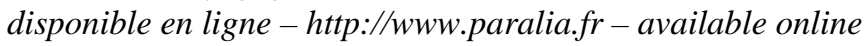

\title{
Protection cathodique des aciers dans le béton armé par courant imposé sur une structure portuaire
}

\author{
Olivier LESIEUTRE ${ }^{1}$, Arnaud MEILLIER ${ }^{1}$, \\ Xavier HALLOPEAU ${ }^{1}$, François TEPLY ${ }^{1}$
}

\section{FREYSSINET,}

Av Napoléon Bonaparte, CS 60002, 92506 Rueil Malmaison, France.

Xavier.hallopeau@freyssinet.com ; Arnaud.meillier@freyssinet.com ;

Olivier.lesieutre@freyssinet.com ; Francois.teply@freyssinet.com

\section{Résumé :}

FREYSSINET réalise à travers le monde des opérations de maintenance des structures par la mise en œuvre des procédés de protection cathodique par courant imposé en neuf comme en réparation.

Le groupe dispose en interne de personnels certifiés suivant la norme EN 15257 ou le standard américain NACE Institute Certifications. Il maitrise les savoirs faire avérés en protection cathodique sur les trois secteurs Béton, Terre, Mer, mais aussi des connaissances approfondies du comportement du matériau béton aux différentes étapes du projet : diagnostic matériaux, études et design, méthodes travaux proprement dits, méthodes logistiques adaptées aux structures portuaires (prise en compte du contexte de l'exploitation des structures et des conditions d'accès particulières).

Les travaux une fois terminés, FREYSSINET peut assurer le suivi des ouvrages protégés par protection cathodique, grâce à des automates de contrôle gérés à distance via le GSM ou la 4G, et rédiger les rapports pour le compte du maitre d'ouvrage, offrant ainsi une solution clé en main.

L'objet de cet article est de décrire de façon technique concise la protection cathodique par courant imposé dans le béton et de présenter un projet récemment mené à terme par le groupe FREYSSINET.

Mots-clés : Protection cathodique, Secteur béton, Courant imposé, Anodes, Béton armé, Béton précontraint, Certification personnel, NF EN 12696, NF EN 15257.

\section{Introduction}

Les ouvrages portuaires en béton armé sont exposés à des conditions agressives de corrosion dues à l'environnement marin (embruns et eau de mer chargés en ions chlorures).

Lorsque ces agents agressifs sont en quantités suffisantes à l'interface acier/béton, les forces expansives générées par les produits de corrosion, et/ou la diminution des sections efficaces d'acier par dissolution de celui-ci, engendrent la dégradation de l'enrobage béton. Les opérations de maintenance et de réparation de béton dites 


\section{Thème 4 - Ouvrages portuaires, offshore et de plaisance}

classiques reposent essentiellement sur la purge et la réparation des bétons dégradés qui amènent à des cycles de réparation rapprochés.

En fonction des dégradations constatées et du taux de chlorures dans le béton, de leur pénétration à cœur et de la difficulté à empêcher leur pénétration à l'avenir, la mise en œuvre d'une protection cathodique par courant imposé en complément des réparations des bétons augmente la pérennité de l'ouvrage sur le moyen à long terme (30 ans et +), offrant ainsi des perspectives optimisées en termes de maintenance et de rénovation des infrastructures.

\section{Rappel sur la corrosion des aciers dans le béton}

En raison de l'alcalinité ( $\mathrm{pH}$ autour de 13) du béton conférée par les solutions basiques contenues dans les pores du béton, l'acier est dans un état de passivité électrochimique. L'acier forme en s'oxydant une couche stable protectrice d'oxydes, diminuant fortement la vitesse de corrosion. Lorsque des agents agressifs sont en quantité suffisante à l'interface acier/béton la couche de passivité peut être altérée soit de manière généralisée soit de manière locale par piqûres. Les principaux agents agressifs responsables de ces phénomènes sont :

- Le dioxyde de carbone $\mathrm{CO}_{2}$ qui par réaction avec la chaux hydratée du béton provoque un abaissement du $\mathrm{pH}$, le phénomène, dit de carbonatation, engendre une corrosion expansive généralisée,

- Les ions chlorures de l'atmosphère marine (embruns et éclaboussures) qui pénètrent dans le réseau capillaire du béton d'enrobage et provoquent une rupture du film de passivité et le développement d'une corrosion dissolvante par piqûre.

Le processus de corrosion des aciers passe par 3 phases: incubation, amorçage et propagation, suivant le modèle TUTTI (1982) illustré sur la figure 1.

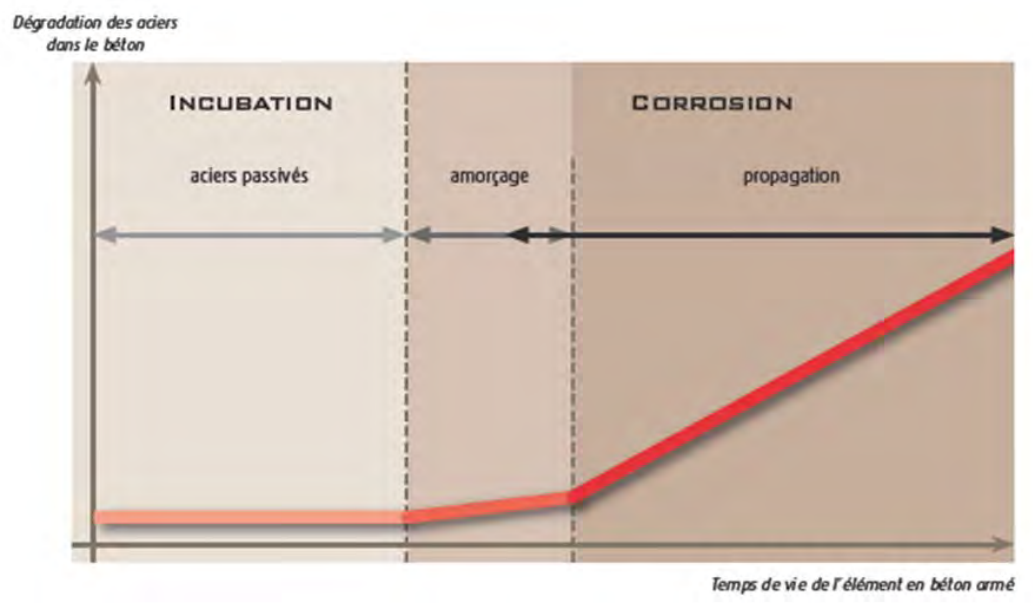

Figure 1. Illustration des 3 phases du phénomène de dégradation du béton armé par le processus de corrosion (TUTTI, 1982). 


\section{XIV ${ }^{\text {èmes }}$ Journées Nationales Génie Côtier - Génie Civil \\ Toulon, 29 juin au $1^{\text {er }}$ juillet 2016}

Pour les structures portuaires, les dégradations des bétons se situent principalement en zone de marnage ou dans la zone d'éclaboussures en fonction de la disponibilité en oxygène à l'interface acier/ béton, car la réduction de l'oxygène est nécessaire pour la formation d'une pile de corrosion. Les zones complétement immergées sont généralement moins concernées, à l'exception de structures "creuses" comprenant un espace interne rempli d'air qui peut entrainer un risque de corrosion par aération différentielle entre la zone aérienne fortement oxygénée qui se comporte comme une cathode et la zone immergée pauvre en oxygène qui se comporte comme une anode, ce risque est aggravé par la teneur en chlorure.

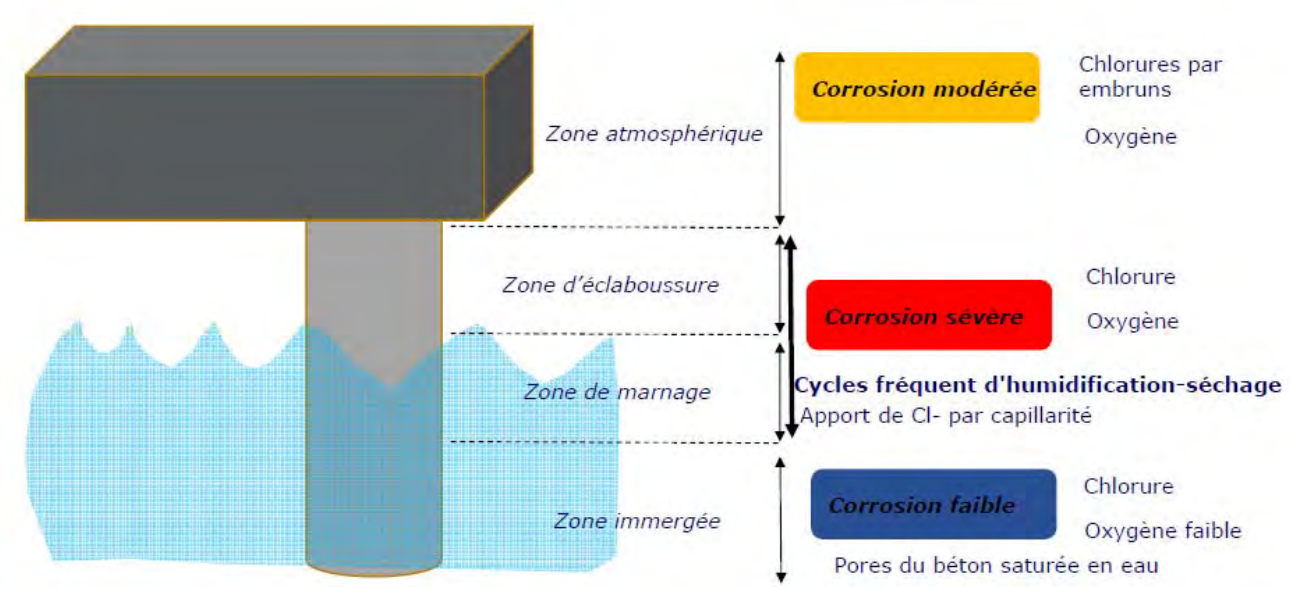

Figure 2. Coupe descriptive des zones de corrosion d'une pile en béton armé dans un environnement marin (voir DAILY, Online).

\section{Principe de la Protection Cathodique par Courant Imposé (PCCI)}

Le principe de la PCCI est de fournir aux aciers plus d'énergie qu'ils n'en ont besoin pour se protéger, et ainsi ramener la corrosion à un niveau considéré comme négligeable. La PCCI implique l'utilisation d'anodes en matériaux nobles (inerte du point de vue électrochimique et stable pour une période de 100 ans) et d'alimentations électriques, dont le débit peut être ajusté en fonction de la demande des aciers à protéger. Cette technique est particulièrement adaptée pour des expositions à fortes teneurs en agents agressifs comme le cas des structures portuaires, et est décrite dans sa globalité par la norme européenne EN 12696.

Un système de protection cathodique par courant imposé tel que mis en œuvre par FREYSSINET sur le Môle des capucins est composé par :

- Un ou plusieurs transformateurs redresseurs automatisés qui produisent le courant nécessaire à la protection des armatures, ils sont équipés de télésurveillance qui permet le monitoring et le contrôle à distance (voir figure 3). 


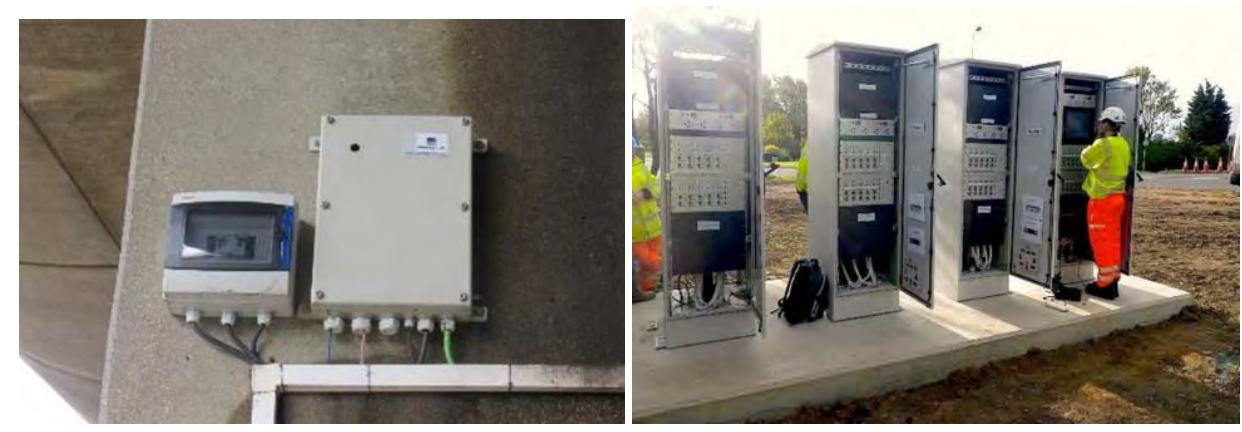

Figure 3. Exemples de transformateurs redresseurs.

- Un circuit anodique qui est relié au redresseur (avec des câbles rouge en cuivre revêtu à double isolation XLPE/XLPE, ramenés au pôle positif de l'alimentation électrique/redresseur), qui diffuse le courant de protection dans le béton; il est principalement constitué par un type d'anode, ou la combinaison de différents types d'anodes décrits ci-après :

a) Les anodes sous forme de treillis fixées sur le béton et recouverte d'un enrobage de $3 \mathrm{~cm}$ (figure 4). Ce système est généralement retenu dans le cadre de projets de réparation par béton projeté, avec des traitements de grandes surfaces.

b)Les anodes de type ruban (figure 5) qui sont placées généralement, pour les structures existantes, dans des saignées dans le béton d'enrobage lorsque celui-ci est en grande partie sain et avec une épaisseur suffisante de $4-5 \mathrm{~cm}$; ou sur une première couche de béton de réparation; ou encore insérées dans la cage d'armatures via des écarteurs plastiques dans le cas d'ouvrage neuf et/ou coulée en place (l'enrobage est dans ce cas de 5 cm minimum Cf. EN 206).

c) Les anodes tubulaires dites discrètes placées dans des forages, chaque anode étant reliée aux autres par un câble anodique titane (figure 6).

Le choix du type d'anodes se fait en fonction des besoins en courant de protection, de la forme des éléments à protéger et des contraintes propres au chantier (réparation (ou non), béton projeté (ou non), difficultés d’accès....).

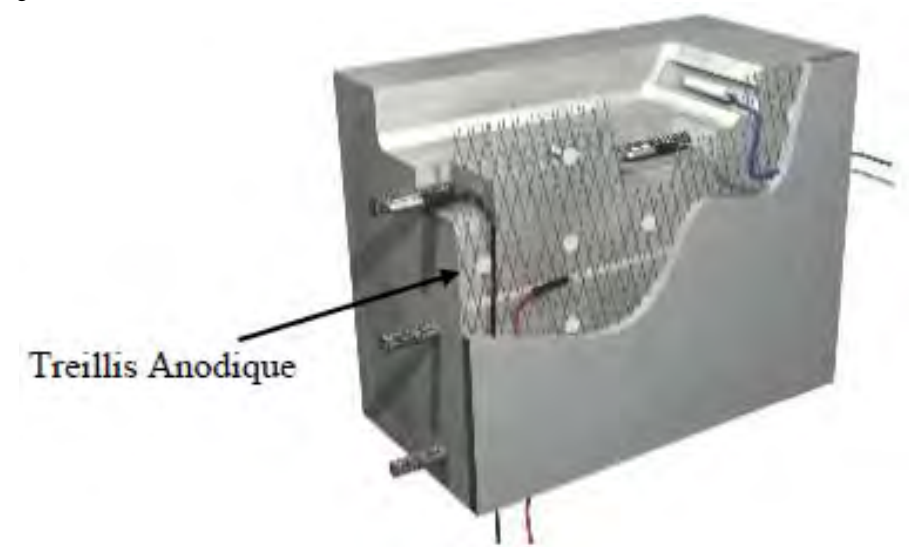

Figure 4. Schéma Anode Treillis titane activé (MESH). 


\section{XIV èmes Journées Nationales Génie Côtier - Génie Civil \\ Toulon, 29 juin au $1^{\text {er }}$ juillet 2016}

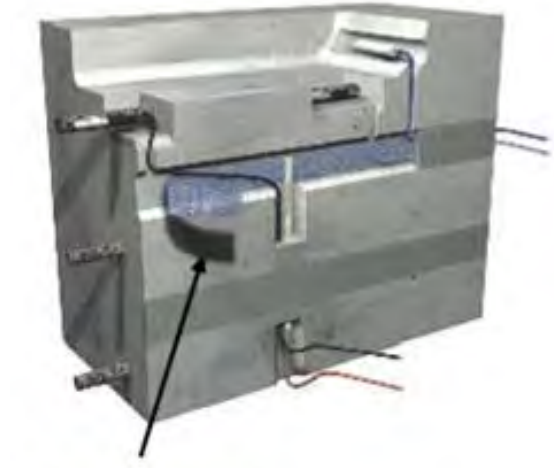

Rubans Anodique

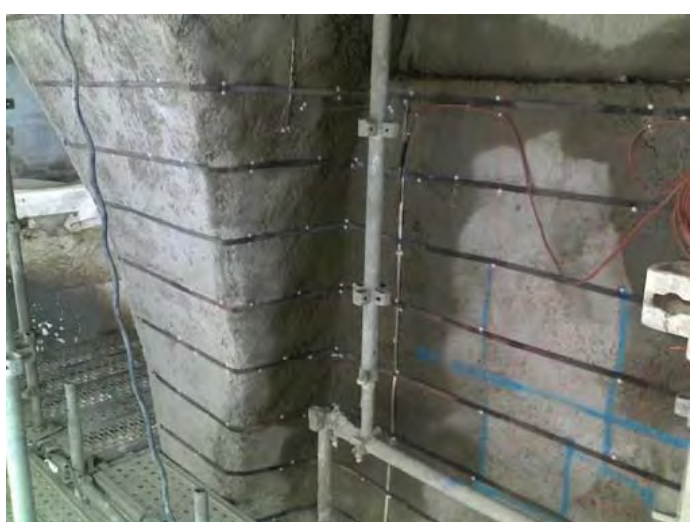

Figure 5. Schéma anode en bande (RIBBON).

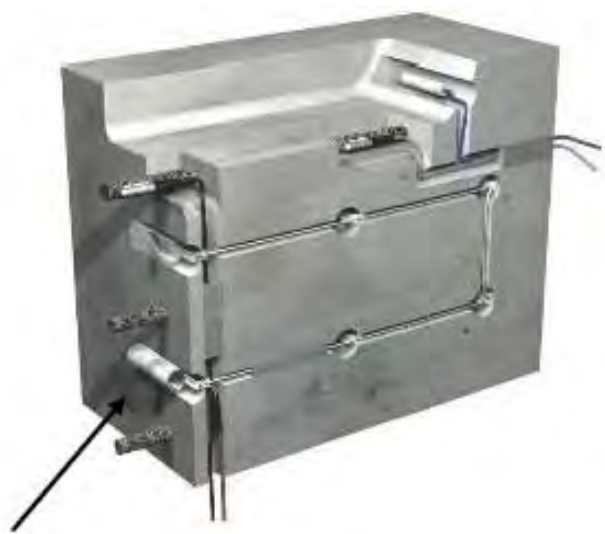

Anode tubulaire

Figure 6. Schéma anodes discrètes forées en titane activé.

- Un circuit cathodique qui est constitué par les armatures à protéger, ces armatures sont connectées par des contacts soudés et isolés à des câbles en cuivre revêtu à double isolation noir XLPE/XLPE, ramenés au pôle négatif de l'alimentation électrique/redresseur.

- Des électrodes de référence et des sondes de dépolarisation installées dans les zones de réparation avec leurs câbles, ramenés à la centrale de mesure. Ces électrodes permettent de mesurer le potentiel électrochimique des aciers et de vérifier les niveaux de protection appliqués (monitoring).

\section{Exemple de structure portuaire : le môle des capucins à Ajaccio (Corse)}

L'ouvrage est composé de 9 structures distinctes. Ces structures ont été construites les unes après les autres de manière contiguë, afin d'augmenter progressivement la capacité d'accueil du môle. Les dates de construction sont comprises entre 1968 et 1990. Des inspections et diagnostics réalisés vers la fin des années 2000 ont mis en évidence une forte corrosion des armatures avec pertes de section et dégradations des bétons sur l'ensemble des éléments. Des travaux de réparation et mise en place d'une protection 


\section{Thème 4 - Ouvrages portuaires, offshore et de plaisance}

cathodique ont été réalisés par le groupement d'entreprise FREYSSINET, APEX, CORSE TRAVAUX et NATALI (Freyssinet mandataire) entre mi 2013 et fin 2015. La plupart des éléments constituant de la structure sont des dalles de compression coulées sur des prédalles avec des poutres en maillage rectangulaire reposant sur des pieux métalliques. Les retombées de poutre limitent à moins d'un mètre la hauteur libre. Il s'agissait d'une des contraintes majeures du chantier (hors maintien en fonctionnement partiel du môle, et météo bien sur....).

Les travaux de réparations ont consisté en une restructuration lourde avec ajout d'aciers de renfort, tant sur les poutres qu'en intrados des dalles, environ 1500 tonnes d'acier et 2500 tonnes de béton projeté par voie sèche ont été mis en œuvre(figure 8). Les opérations préalables de purge du béton dégradé ont permis d'effectuer les tests de continuité électrique des aciers en place (dans les zones d'influence de la PCCI), de la rétablir en cas de besoin, et d'établir celle-ci entre les nouveaux aciers de renforts et les anciens aciers initiaux.

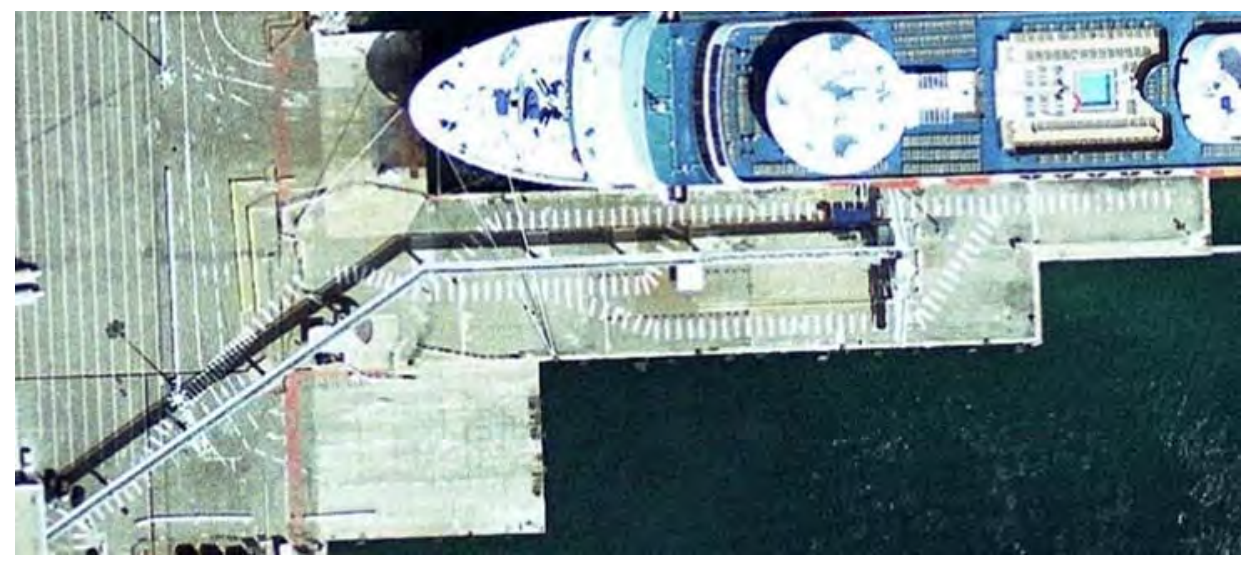

Figure 7. Photo aérienne du môle des capucins Ajaccio.
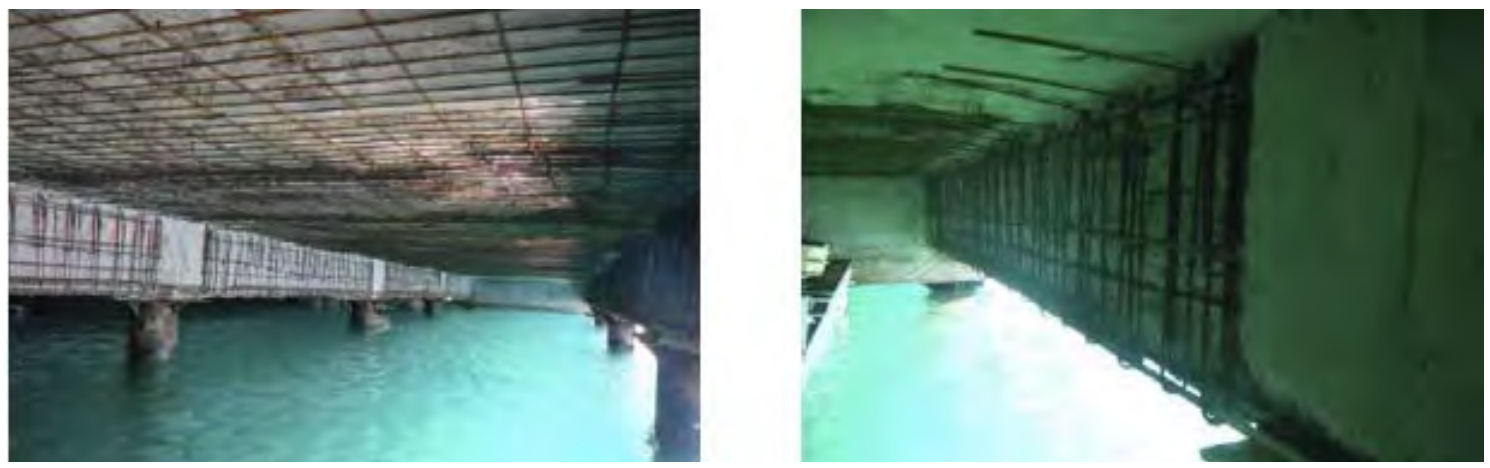

Figure 8. Aciers de renforts mis en œuvre avant le béton projeté par voie sèche.

En ce qui concerne les phases de conception, de mise en œuvre et de suivi de la protection cathodique dans le béton armé, les personnels impliqués sur cette opération étaient certifiés dans le secteur béton : 


\section{XIV èmes Journées Nationales Génie Côtier - Génie Civil \\ Toulon, 29 juin au $1^{\text {er }}$ juillet 2016}

- niveau 2 minimum selon la norme EN 15257 (ou certifiés selon la NACE Américaine au level 4) pour l'ingénierie support, et niveau 1 pour les personnels de production et l'encadrement du chantier.

En effet le personnel impliqué dans la conception a du faire le choix du positionnement des points de mesurage qui a été un des vrais problèmes de ce chantier; avec une réflexion sur la pertinence du positionnement de ceux-ci en fonction de la présence de précontrainte et de la recherche du potentiel vrai, tant des anciens aciers que des nouveaux qui constituent le renfort. Le système de protection cathodique par courant imposé a été conçu et mis en place comme tel :

- définition de 4 zones principales nommées MCPC01, MCPC02, MCPC03 et MCPC08 (figure 9) qui correspondent aux différents ouvrages rajoutés dans le temps qui constituent le môle actuel, réparties en 20 zones de protection cathodique indépendantes (voies de puissance dédiées),

- mise en œuvre de 160 points de mesurage (80 électrodes de références couplées avec 80 sondes dépolarisation Titane).

- Mise en œuvre d'environ 3600 anodes forées en titane activé pour la protection des poutres, permettant une polarisation à cœur des éléments porteurs filants, suite aux résultats d'essais pilotes qui ont permis de vérifier les hypothèses de calcul, (notamment l'inter-distance entre anodes discrètes forées), ainsi que le calcul de la chute de tension dans le circuit anodique en fonction des variations de longueurs de chapelets d'anodes forées,

- Mise en œuvre d'environ $3000 \mathrm{~m}^{2}$ de treillis titane activé pour la protection des sous faces des dalles en conjonction des travaux de béton projeté (figure 10), avec la prise en compte de la présence de câbles de précontrainte dans les éléments structurels, qui amène à une gestion des courants de protection par un contrôle en auto-potentiel,

- Mise en place d'une télégestion et télé-contrôle par voie GSM.

- Conception et mise en œuvre d'une protection kéraunique (protection foudre) des installations, de façon à les rendre suffisamment robustes au regard des conditions environnementales,

- prise en compte des courants vagabonds éventuels.
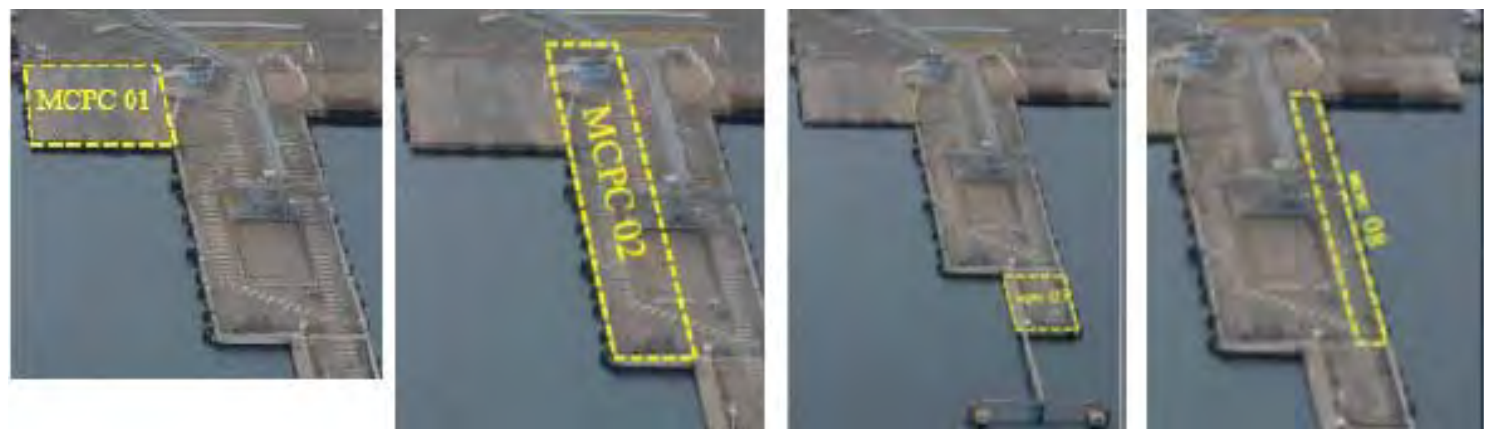

Figure 9. De gauche à droite : MCPC01, МCPC02, MCPC03, МСРC08. 


\section{Thème 4-Ouvrages portuaires, offshore et de plaisance}
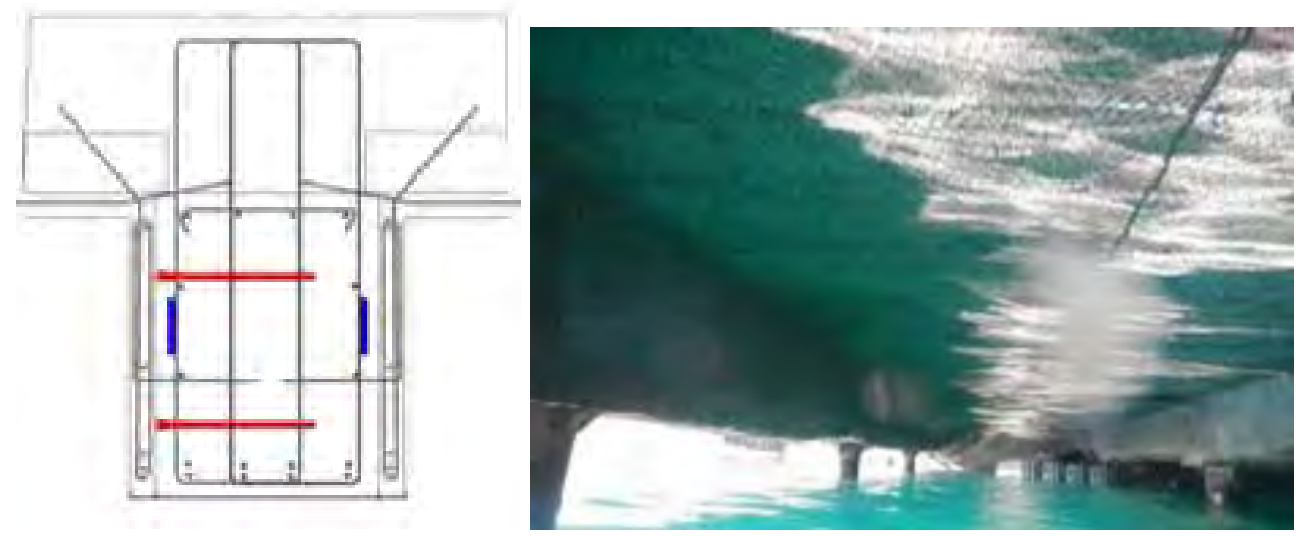

Figure 10. Mise en œuvre des anodes forées discrètes dans les poutres et du MESH en intrados de dalle avant réalisation béton projeté voie sèche.

Le chantier (comprenant également un autre ouvrage) a duré au total presque 30 mois, mobilisé un effectif moyen d'une dizaine de personnes pour un cumul d'environ 30000 heures de travail.

\section{Conclusions perspectives}

La protection cathodique dans le béton armé en conjonction avec les travaux de réparation est une technique mature et normalisée, qui offre des vraies perspectives d'optimisation dans la stratégie de maintenance pour les autorités portuaires, en rallongeant les cycles de réparation et assurant un retour d'investissement sur le long terme. La mise en place d'une protection cathodique dès la construction d'un ouvrage peut aussi se concevoir (on parle alors de "prévention" cathodique) : elle permet d'augmenter de façon significative la durabilité des bétons dès le début de vie de la structure, à titre d'exemple : elle est actuellement mise en place sur les 48 piles du viaduc de $5 \mathrm{~km}$ et du viaduc de la grande chaloupe, sur la nouvelle route du littoral à La Réunion.

\section{Références bibliographiques}

DAILY S.F. (Online). Using cathodic protection to control corrosion of reinforced concrete structures in marine environments. Corrpro Technical Library, Paper CP 49. Available from URL : http://www.corrpro.com/Resources/Technical-Papers

TUTTI K. (1982). Corrosion of steel in concrete. Swedish Cement and Concrete Research Institute, Stockholm.

NF EN 12696 (2012) Protection cathodique de l'acier dans le béton.

NF EN 15257 (2007). Protection cathodique - Niveaux de compétence des personnes en protection cathodique - Base pour un dispositif particulier de certification. 